\title{
The action of red wine and purple grape juice on vascular reactivity is independent of plasma lipids in hypercholesterolemic patients
}

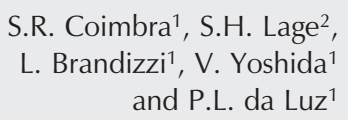

\author{
${ }^{1}$ Unidade de Ateroesclerose, ${ }^{2}$ Unidade de Terapia Intensiva, \\ Instituto do Coração, Hospital das Clínicas, Faculdade de Medicina, \\ Universidade de São Paulo, São Paulo, SP, Brasil
}

\begin{abstract}
Correspondence
P.L. da Luz

Unidade de Ateroesclerose

InCor, HC, FM, USP

Av. Dr. Eneas C. Aguiar, 44

05403-000 São Paulo, SP

Brasil

Fax: +55-11-3069-5447

E-mail: daluzp@incor.usp.br
\end{abstract}

Research supported by FAPESP (No. 1998/10576-5) and Fundação Zerbini. ....................

Received May 27, 2004 Accepted May 20, 2005

\begin{abstract}
Although red wine (RW) reduces cardiovascular risk, the mechanisms underlying the effect have not been identified. Correction of endothelial dysfunction by RW flavonoids could be one mechanism. We measured brachial artery reactivity by high-resolution ultrasonography, plasma lipids, glucose, adhesion molecules (ICAM-1 and VCAM), and platelet function in 16 hypercholesterolemic individuals $(8 \mathrm{men}$ and 8 women; mean age $51.6 \pm 8.1$ years) without other risk factors. Twenty-four normal subjects were used as controls for vascular reactivity. Subjects randomly received RW, $250 \mathrm{ml} /$ day, or purple grape juice (GJ), $500 \mathrm{ml} /$ day, for 14 days with an equal wash-out period. At baseline, all 16 subjects were hypercholesterolemic (mean $\mathrm{LDL}=181.0 \pm 28.7 \mathrm{mg} / \mathrm{dl}$ ) but HDL, triglycerides, glucose, adhesion molecules, and platelet function were within normal limits. Brachial artery flow-mediated dilation was significantly decreased compared to controls $(9.0 \pm 7.1$ vs $12.1 \pm 4.5 \% ; \mathrm{P}<0.05)$ and increased with both GJ $(10.1 \pm 7.1$ before $v s 16.9 \pm 6.7 \%$ after: $\mathrm{P}<0.05)$ and RW $(10.1 \pm$ 6.4 before vs $15.6 \pm 4.6 \%$ after; $\mathrm{P}<0.05)$. RW, but not GJ, also significantly increased endothelium-independent vasodilation (17.0 \pm 8.6 before $v$ s $23.0 \pm 12.0 \%$ after; $\mathrm{P}<0.01$ ). GJ reduced ICAM- 1 but not VCAM and RW had no effect on either molecule. No significant alterations were observed in plasma lipids, glucose or platelet aggregability with RW or GJ. Both RW and GJ similarly improved flow-mediated dilation, but RW also enhanced endothelium-independent vasodilation in hypercholesterolemic patients despite the increased plasma cholesterol. Thus, we conclude that GJ may protect against coronary artery disease without the additional negative effects of alcohol despite the gender.
\end{abstract}

\section{Introduction}

Moderate drinking is associated with a decreased risk of myocardial infarction, stroke, and cardiac death $(1,2)$, as well as
Key words

- Atherosclerosis

- Endothelial function

- Flavonoids

- Alcohol

- Red wine and grape juice

- Brachial artery dilation 
platelets, some studies indicate that the greatest protection is due to red wine (RW), rather than beer or spirits (8-10). This suggests that phenolic acids, polyphenols, and or flavonoids may confer additional benefits.

Flavonoids are polyphenol derivatives of 2-phenyl-1-benzopyran-4-1 that are present in fruits, vegetables, nuts, and seeds (11). In epidemiological studies, flavonoid intake is associated with a reduced risk of coronary events $(11,12)$. In an experimental study (13) we showed that both RW and nonalcoholic wine products can prevent plaque formation in hypercholesterolemic rabbits despite significant increases in low-density lipoprotein (LDL). Apoprotein E (Apo E)deficient mice also showed reduced atherosclerosis progression when fed RW (14). RW also causes monocyte chemotactic protein-1 expression blockade and reduced neointimal hyperplasia after balloon injury in rabbits (15), inhibition of smooth muscle cell proliferation and of cyclin A expression (16), as well as inhibition of the plateletderived growth factor receptor by RW flavonoids in cultured smooth muscle cells (17) - all of them factors contributing to atherogenesis.

More recent evidence (18) indicated that RW, but not vodka, inhibited nuclear factor$\kappa \mathrm{B}$ expression in leukocytes of human volunteers fed a high fat diet. Furthermore, RW phenols blocked endothelin-1 production (19) and induced endothelial nitric oxide synthase (eNOS) expression in cultured endothelial cells (20).

In vitro, the flavonoid components of RW and purple grape juice (GJ) induce endothelium-dependent vasodilation of arterial rings (21), a phenomenon mediated by the nitric oxide cyclic guanosine monophosphate (NO-cGMP) pathway (22). Although improved endothelial function and inhibition of platelet aggregation are potential mechanisms by which RW flavonoids may reduce cardiovascular risk in hypercholesterolemic patients, the in vivo effects of RW and GJ on these parameters are not well known. Despite favorable clinical and experimental evidence, recommendation for wine or any alcohol drinking is controversial, due to well known untoward side effects of alcohol.

Hence we decided to compare RW and GJ in humans to determine whether the benefits of polyphenols could be achieved without the undesirable side effects of alcohol. The specific purpose of this study was to compare the effects of RW and GJ on arterial reactivity, platelet aggregability, adhesion molecules, glucose, and plasma lipids in hypercholesterolemic subjects.

\section{Patients and Methods}

\section{Patients}

Sixteen healthy adult subjects with isolated hypercholesterolemia were enrolled. Exclusion criteria included smoking, diabetes mellitus, hypertension, lipid-lowering therapy, and obesity (body mass index $>26$ ). In order to establish normal values for vascular reactivity, 24 healthy individuals were also studied. Study subjects were not allowed to consume fruit products, tea, or alcoholic beverages during the study. They were encouraged not to change their diet otherwise and to keep a diary of daily intake of all food and beverages which was reviewed to assure dietary compliance. A complete medical history was obtained and physical examination was carried out at study entry. The study was approved by the Hospital Ethics Committee. Each subject gave written informed consent to participate in the study after a thorough explanation of the study design and protocol. Subjects were provided with commercial purple GJ and instructed to drink $500 \mathrm{ml} /$ day, and with RW Pinot Noir (Aurora Company, Bento Gonçalves, RS, Brazil) and instructed to drink $250 \mathrm{ml} /$ day for 14 days according to the study schedule. 


\section{Brachial artery reactivity}

Brachial artery (BA) reactivity studies were always performed in the morning after a 10-min rest in a temperature-controlled room $\left(20^{\circ}\right.$ to $\left.25^{\circ} \mathrm{C}\right)$. The diameter of the left $\mathrm{BA}$ and baseline forearm blood flow velocity were measured with a $7.5-\mathrm{MHz}$ linear array vascular ultrasound transducer part of an APOGEE 800 plus ATL ultrasound system (ATL Ultrasound, Bothell, WA, USA). For the evaluation of endothelial-dependent reactivity (23-25) increased forearm blood flow was induced by inflation of a pneumatic blood pressure tourniquet placed around the widest part of the forearm to a systolic pressure of $250 \mathrm{mmHg}$, followed by deflation after $5 \mathrm{~min}$. The first five blood flow scans were obtained continuously immediately thereafter and blood flow velocities and BA diameters were measured again after $1 \mathrm{~min}$. Twenty minutes were allowed for vessel recovery, and repeat resting BA diameter and blood flow scans were obtained. For endothelial-independent evaluation, sublingual isosorbide dinitrate (5 mg) was administered and final scans were performed after $5 \mathrm{~min}$. A single-lead ECG was monitored throughout the study. Blood pressure was measured in the right upper arm before the first scan, before administration of sublingual nitroglycerin, and every 5 min thereafter until it returned to baseline.

Ultrasound images were recorded in VHS. The BA was imaged 2 to $15 \mathrm{~cm}$ above the elbow and scanned in longitudinal section with the focus zone set to the depth of the near wall. During image acquisition, anatomic landmarks such as fascial planes were noted to help maintain the same image of the artery throughout the study. Depth and gain settings were used to optimize the images of the lumen/arterial wall interface. Vessel diameters were measured using a software program, developed at InCor, a semi-automatic approach to measure artery diameter based on active contour technique improved by multiresolution analysis. The operator selected a region of interest $(1 \mathrm{~cm}$ in longitudinal diameter) in six series of brachial images obtained from $b$-mode ultrasound. The distance between near wall media-adventitia and far wall media-adventitia was obtained for all images and the mean value was calculated during diastole (26).

Flow-mediated vasodilation (FMD) was calculated as the ratio of the BA diameter after reactive hyperemia to the baseline diameter, expressed as a percent change. Nitroglycerin-mediated vasodilation (NTGD) was calculated in an analogous fashion.

The intraobserver reliability for measurement of BA diameters was 0.987. This value reflects the interclass correlation coefficient across all readings and all conditions (baseline, reactive hyperemia, prenitroglycerin, and postnitroglycerin).

\section{Lipids and platelet aggregation}

Plasma samples were collected in EDTA after a 12-h fast by venipuncture performed on the same day as the BA studies.

Plasma total cholesterol and triglycerides were determined using enzymatic methods (Roche Laboratories, Basel, Switzerland) (27). HDL-cholesterol was determined by the same method as for total cholesterol, and after chemical precipitation of apolipoprotein B100-containing lipoproteins with $\mathrm{MgCl}$ and phosphotungstic acid. VLDL-cholesterol and LDL-cholesterol were estimated by the Friedewald formula. Apo B and Apo AI were also determined by immunoturbidimetry (Roche). All lipid and apolipoprotein determinations were performed automatically using a COBAS-MIRA analyzer (Roche). Lipoprotein (a) (Lp(a)) was measured by immunoprecipitation analyses and immunoturbidimetric methods. Platelet aggregation was estimated by the Born and Cross turbidimetric methods. The results are reported as normal, increased or decreased platelet aggregation. 


\section{Adhesion molecules}

Serum was separated from whole blood following centrifugation in a benchtop centrifuge for $15 \mathrm{~min}$ at $750 \mathrm{~g}$ and was stored at $-80^{\circ} \mathrm{C}$ until assayed. Serum levels of intercellular adhesion molecule 1 (h-sICAM-1 ELISA, anti-sICAM-1: monoclonal antibody from murine, parameter R\&D Systems, Minneapolis, MN, USA) and vascular cell adhesion molecule (h-sVCAM-1 ELISA, antisVCAM-1 biotin, monoclonal antibody from mouse, clone M-1.G11-B1, and antisVCAM-1 peroxidase, monoclonal antibody from mouse, clone M-DDV-1 fab fragments) were determined by ELISA.

\section{Protocol}

Sixteen subjects were randomized to receive either $500 \mathrm{ml} /$ day GJ or $250 \mathrm{ml} /$ day RW for 14 days as first treatment; a wash-out period of equal duration was then observed, followed by the second treatment modality.

Patients were examined every 2 weeks after an overnight 12-h fast. Endothelial function was assessed four times: 1) at baseline, 2) at the end of the first treatment period, 3) after a 14-day washout, and 4) after the second treatment period. At all 4 points, blood was collected and vital signs were measured with automated monitor after 20 min of rest.

\section{Statistical analysis}

Continuous variables are reported as means \pm SD. Changes in FMD and NTGD are described as means with $95 \%$ confidence. FMD and NTGB were compared by ANOVA with repeated measures and categorical variables were compared by the Fisher test. The level of significance was set at $\mathrm{P} \leq 0.05$ for all analyses.

Table 1. Characteristics of hypercholesterolemic patients at baseline and after the ingestion of red wine or grape juice.

\begin{tabular}{|c|c|c|c|c|}
\hline & \multicolumn{2}{|c|}{ Red wine } & \multicolumn{2}{|c|}{ Grape juice } \\
\hline & Before & After & Before & After \\
\hline Body mass index $\left(\mathrm{kg} / \mathrm{m}^{2}\right)$ & $24.8 \pm 1.5$ & $24.8 \pm 1.4$ & $24.8 \pm 1.5$ & $24.8 \pm 1.2$ \\
\hline Heart rate $(\mathrm{bpm})$ & $62.1 \pm 8.0$ & $62.9 \pm 9.0$ & $60.6 \pm 6.3$ & $61.4 \pm 8.5$ \\
\hline Systolic blood pressure (mmHg) & $123.0 \pm 9.9$ & $128.2 \pm 10.5$ & $122.0 \pm 10.3$ & $124.7 \pm 12.0$ \\
\hline Diastolic blood pressure $(\mathrm{mmHg})$ & $76.6 \pm 5.3$ & $77.8 \pm 6.2$ & $74.5 \pm 5.6$ & $78.2 \pm 7.0$ \\
\hline Mean blood pressure (mmHg) & $92.1 \pm 6.1$ & $94.6 \pm 4.7$ & $90.3 \pm 6.6$ & $93.7 \pm 8.4$ \\
\hline Total cholesterol (mg/dl) & $252.7 \pm 35.1$ & $258.0 \pm 42.2$ & $259.0 \pm 35.1$ & $253.0 \pm 40.4$ \\
\hline LDL cholesterol (mg/dl) & $175.1 \pm 28.7$ & $178.2 \pm 32.7$ & $179.0 \pm 27.7$ & $176.0 \pm 31.4$ \\
\hline HDL cholesterol (mg/dl) & $55.6 \pm 17.7$ & $55.9 \pm 17.0$ & $56.0 \pm 15.9$ & $53.0 \pm 12.5$ \\
\hline Triglycerides (mg/dl) & $110.1 \pm 40.6$ & $118.2 \pm 57.9$ & $124.0 \pm 46.8$ & $117.0 \pm 50.9$ \\
\hline Lp(a) (mg/dl) & $35.5 \pm 34.5$ & $26.2 \pm 18.8$ & $35.5 \pm 34.5$ & $30.5 \pm 24.5$ \\
\hline Apo A (mg/dl) & $1.5 \pm 0.3$ & $1.5 \pm 0.3$ & $1.5 \pm 0.3$ & $1.5 \pm 0.3$ \\
\hline Apo B (mg/dl) & $1.3 \pm 0.3$ & $1.4 \pm 0.2$ & $1.3 \pm 0.3$ & $1.5 \pm 0.2$ \\
\hline Glucose (mg/dl) & $88.6 \pm 6.5$ & $93.0 \pm 8.6$ & $90.0 \pm 7.3$ & $90.0 \pm 6.9$ \\
\hline VCAM-1 (ng/ml) & $363.3 \pm 127.3$ & $380.7 \pm 81.8$ & $384.7 \pm 105.4$ & $363.8 \pm 82.5$ \\
\hline ICAM-1 (ng/ml) & $140.6 \pm 46.6$ & $130.6 \pm 55.5$ & $146.8 \pm 44.9$ & $115.9 \pm 21.8^{*}$ \\
\hline
\end{tabular}

Data are reported as means $\pm S D(N=16)$. $L p(a)=$ lipoprotein $a ; A p o=$ apolipoprotein; $V C A M-1=$ vascular cell adhesion molecule 1 ; ICAM-1 = intercellular adhesion molecule 1.

${ }^{*} \mathrm{P}<0.05$ compared to baseline grape juice (Fisher test). 


\section{Results}

\section{Baseline characteristics}

For the specific purpose of establishing normal values for vascular reactivity for our laboratory, 24 healthy subjects were studied. Their mean age was $42.6 \pm 7.1$ years, and blood pressure was $124 / 80 \pm 12 / 5 \mathrm{mmHg}$, total cholesterol was $180 \pm 9.1 \mathrm{mg} / \mathrm{dl}$, LDL $111 \pm 7.9 \mathrm{mg} / \mathrm{dl}$, HDL $53 \pm 2.2 \mathrm{mg} / \mathrm{dl}$, triglycerides were $155 \pm 10.1 \mathrm{mg} / \mathrm{dl}$, and fasting glucose was $89 \pm 12 \mathrm{mg} / \mathrm{dl}$. The brachial artery diameter averaged $3.55 \pm 0.6 \mathrm{~mm}$, FMD was $12.1 \pm 4.5 \%$ and NTGD was 16.6 $\pm 9.5 \%$. These subjects were not included in the RW/GJ study.

Of the 16 randomized subjects 8 were males and 8 were females. Their average age was $51.6 \pm 8.1(\mathrm{SD})$ years. All subjects were hypercholesterolemic and none was receiving lipid-lowering therapy. Table 1 describes the demographic data of the subjects.

Comparisons were made at different times in the same patients and between treatment groups. There were no statistically significant differences between baseline, pretreatment and washout values for any parameter. There was no interaction between beverage order and treatment effects $(\mathrm{P}=0.93)$.

\section{Brachial artery reactivity}

Red wine effects. In the 16 hypercholesterolemic subjects the mean baseline BA diameter was significantly increased compared with control $(3.8 \pm 0.7$ vs $3.55 \pm 0.6$ $\mathrm{mm}, \mathrm{P}<0.05)$. After 2 weeks of RW, the mean baseline diameter remained unchanged ( $3.9 \pm 0.8$ vs $3.8 \pm 0.7 \mathrm{~mm}, \mathrm{P}=\mathrm{ns})$.

Baseline FMD was significantly impaired in hypercholesterolemic subjects compared with controls $(9.0 \pm 7.1$ vs $12.1 \pm 4.5 \%, \mathrm{P}<$ 0.05). After RW, FMD increased significantly $(10.1 \pm 6.4$ vs $15.6 \pm 4.6 \%, \mathrm{P}<0.05)$ in hypercholesterolemic subjects (Figure 1A). Red wine had no effect on the intensity of reactive hyperemic blood velocity (data not shown).

At baseline, NTGD did not differ between hypercholesterolemic and control subjects $(18.9 \pm 7.6$ vs $16.6 \pm 9.5 \%, \mathrm{P}=\mathrm{ns})$. However, after RW, NTGD increased from $16.6 \pm 9.5$ to $23.0 \pm 12.0 \%, \mathrm{P}<0.05$ (Figure 1A).

These effects on vascular reactivity occurred in the absence of any significant changes in heart rate and blood pressure, as shown in Table 1.

Grape juice. Similar to RW, the mean baseline BA diameter of hypercholesterolemic subjects remained unchanged after 2 weeks of GJ consumption $(3.9 \pm 0.8$ vs $3.8 \pm$ $0.7 \mathrm{~mm}$; $\mathrm{P}=\mathrm{ns}$ ). On the other hand, in hypercholesterolemic subjects FMD increased significantly $(10.9 \pm 7.4$ vs $16.9 \pm$ $6.7 \%, \mathrm{P}<0.05$ ) with GJ (Figure 1B). GJ had no effect on the intensity of reactive hyperemic blood velocity (data not shown).

In contrast, NTGD remained unchanged $(19.8 \pm 8.8 v s 18.0 \pm 9.4 \%, \mathrm{P}=\mathrm{ns})$ after GJ (Figure 1B). These effects were not accompanied by any significant changes in heart rate and systolic, diastolic or mean blood pressure (Table 1).

A comparison of the final FMD values in both RW and GJ treatments showed no sig-
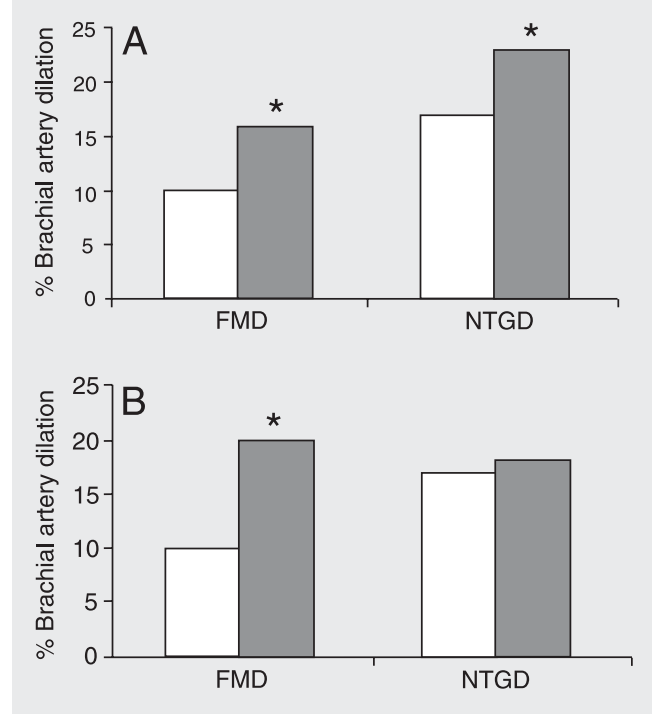

Figure 1. Effect of ingestion of red wine (RW) and grape juice (GJ) on flow-mediated dilation and endothelium-dependent dilation. Twelve hypercholesterolemic patients received $250 \mathrm{ml}$ $\mathrm{RW} /$ day (A) or $500 \mathrm{ml} \mathrm{GJ/day}$ (B) for 14 days. FMD = flowmediated dilation; NTGD = nitroglycerin-mediated dilation. Open columns = before treatment; filled columns $=$ after treatment. ${ }^{*} \mathrm{P} \leq 0.05$ compared to before treatment (ANOVA). 
nificant difference (unpaired $t$-test), indicating that the magnitude of response to both treatments was similar.

\section{Lipid and glucose values}

As shown in Table 1 , subjects were clearly hypercholesterolemic, but triglycerides, HDL-cholesterol and glucose levels were normal. Also, Apo A, Apo B, and Lp(a) were normal. After RW or GJ no effect on fasting plasma lipids, Lp(a), Apo A, Apo B, or glucose was observed (Table 1).

\section{Adhesion molecules}

At baseline, mean adhesion molecule values were within normal ranges and RW had no effect on VCAM-1 or ICAM-1 (Table 1). In contrast to RW, GJ ingestion significantly decreased ICAM-1, but had no effect on VCAM-1.

\section{Platelet aggregation}

At baseline, platelet aggregation was normal in 12 subjects, decreased in 3 and increased in 1. Neither RW nor GJ produced any significant change in platelet aggregation.

\section{Discussion}

This study demonstrates comparable improvement in arterial endothelial dilation after short-term ingestion of both GJ and RW in hypercholesterolemic individuals, without affecting plasma lipids, glucose levels or platelet aggregability. RW, but not GJ, also increased endothelium-independent vasodilation. In addition, GJ reduced ICAM-1 but did not affect VCAM. Since endothelial dysfunction is an early event in atherosclerosis (28) and may be of considerable prognostic value $(29,30)$, these findings may be relevant for vascular protection in patients at risk.

Since FMD increments were similar in
RW and GJ, such improvement cannot be attributed to alcohol, nor can it be attributed to lipid, glucose or platelet effects, because none changed significantly. Therefore, the improvement in endothelial function is most probably due to other substances present in both RW and GJ. Although various chemicals are present in RW and GJ, several lines of evidence point to flavonoids as the most likely to play a role in vascular function.

In vitro the NO-cGMP pathway mediates the endothelium-dependent vasodilating effects of RW and GJ. Ethanol, at the same concentration as contained in red and white wines, does not cause vasorelaxation (31). RW or extracts obtained from it caused endothelium-dependent, NO-mediated vasorelaxation of rat (32) or rabbit (33) aorta preconstricted with norepinephrine. Also, in human coronary arteries and rat aortic rings in vitro, short-term incubation with RW induced FMD and increased vascular cGMP content (31). These effects were abolished after endothelial denudation and reversed by NOS inhibition. Ethanol did not affect vascular tension or cGMP content.

These rapid effects of RW are likely to involve an acute activation of eNOS. Wallerath et al. (20) provided evidence that RW can stimulate the expression of the eNOS gene leading to enhanced production of NO. This upregulation was seen in both EA.hy 926 cells and primary human umbilical vein endothelial cells; such phenomenon is mediated in part by increases in $\mathrm{Ca}^{2+}$ in endothelial cells (34).

Flavonoids may also affect endothelin-1 production. Corder et al. (19) found that RW polyphenols decreased ET-1 synthesis in cultured bovine aortic endothelial cells by suppressing transcription of the ET-1 gene.

In human studies, Stein et al. (35) showed that short-term ingestion of purple GJ improves FMD and reduces LDL susceptibility to oxidation in coronary patients. On the other hand, Hashimoto et al. (36) demonstrated that endothelium-dependent vasodi- 
lation improves after acute intake (120 min) of RW or RW without alcohol in men. But endothelium-independent vasodilation remained unchanged. In contrast, the present study showed that endothelium-independent vasodilation also improves after RW. Therefore, not only endothelial but also smooth muscle cell reactivity function is improved by RW. The mechanisms underlying the latter effect are not clear. One possible explanation is the activation of the parasympathetic nervous system and relative inactivation of the sympathetic nervous system which are known to occur after alcohol consumption (37). Regarding the overall effects, the actions of flavonoids and ethanol on NO production can be invoked. Thus, Matsuo et al. (38) showed an increased acute production of NO with RW and ethanol in the plasma of healthy subjects using diaminofluoresceins as new selective fluorescent indicators.

Hence, the most plausible mechanism responsible for the observed improvement in endothelium-dependent vasodilation is the action of flavonoids. On the one hand, they can decrease endothelin production which may prevent vasoconstriction and on the other, they increase NO production by endothelial cells, thus facilitating vasodilation. Regarding endothelium-independent vasodilation, the mechanisms remain speculative.

It is noteworthy that these vasodilatory effects occurred independently of changes in lipids, glucose or platelet aggregation. This supports the hypothesis of flavonoid mechanisms. Our findings regarding lipids are consistent with those of Hayek et al. (14), who also did not find changes in HDL or LDL after giving RW or the polyphenols quercetin or catechin to mice. However, prolonged alcohol ingestion is known to increase HDL in humans (39); thus, the absence of an RW effect on HDL in the present investigation might have been due to the short period of administration.

The present study also provides evidence that GJ, but not RW, can diminish circulating ICAM-1, perhaps because phenolic compounds can suppress cytokine-induced activation of nuclear factor $\mathrm{\kappa B}$ and subsequent expression of adhesion molecules. However, VCAM-1 was not significantly affected by GJ. The reasons for this selective effect of GJ on ICAM-1 remain unknown.

\section{Limitations}

Although the number of subjects in this study was relatively small, the BA ultrasonic technique for evaluating endothelial function is very sensitive and reproducible, and each subject is used as his own control. The sample size was based on published nomograms for interventions assessed by this technique. Because of the short duration of this study, it is also not known whether the observed vasorelaxing properties of GJ and RW would persist with chronic ingestion. No direct measurement of flavonoids was made; therefore, their influence on reactive vascular is only deductive.

We have shown that short-term ingestion of GJ and RW improves endothelial function and only GJ reduces ICAM-1 in hypercholesterolemic patients. Also, endotheliumindependent vasodilation was increased by RW. Improved endothelium-dependent vasodilation is a potential mechanism by which flavonoids in purple grape products may prevent cardiovascular events, as suggested by several epidemiological studies (40). Of particular interest is the demonstration that the improvement in vascular function can be obtained without alcohol. This is of potential clinical relevance for certain sub-groups of patients at risk of cardiovascular events, for whom even moderate alcohol intake is not advisable.

\section{Acknowledgments}

We thank Silvia Furtado for secretarial assistance. 


\section{References}

1. Renaud S \& de Lorgeril M (1992). Wine, alcohol, platelets, and the French paradox for coronary heart disease. Lancet, 339: 15231526.

2. St Leger AS, Cochrane AL \& Moore F (1979). Factors associated with cardiac mortality in developed countries with particular reference to the consumption of wine. Lancet, 1: 1017-1020.

3. Orgogozo JM, Dartigues JE, Lafont S et al. (1997). Wine consumption and dementia in the elderly: a prospective community study in the Bordeaux area. Revue Neurologique, 153: 185-192.

4. Leibovici D, Ritchie K, Ledesert B et al. (1999). The effects of wine and tobacco consumption on cognitive performance in the elderly: a longitudinal study of relative risk. International Journal of Epidemiology, 28: 77-81.

5. MukamaL KJ, Longstreth Jr WT, Mittleman MA et al. (2001). Alcohol consumption and subclinical findings on magnetic resonance imaging of the brain in older adults: the Cardiovascular Health Study. Stroke, 32: 1939-1946.

6. Obisesan TO, Hirsch R, Kosoko O et al. (1998). Moderate wine consumption is associated with decreased odds of developing agerelated macular degeneration in NHANES-1. Journal of the American Geriatric Society, 46: 1-7.

7. Klein R, Klein BE \& Moss SE (1989). The Wisconsin epidemiological study of diabetic retinopathy: a review. Diabetes/Metabolism Reviews, 5: 559-570.

8. Gronbaek M, Deis A, Sorensen TI et al. (1995). Mortality associated with moderate intakes of wine, beer, or spirits. British Medical Journal (Clinical Research), 310: 1165-1169.

9. Truelsen T, Gronbaek M, Schnohr P et al. (1998). Intake of beer, wine, and spirits and risk of stroke: The Copenhagen City Heart Study. Stroke, 29: 2467-2472.

10. Rimm EB, Klatsky A, Grobbee D et al. (1996). Review of moderate alcohol consumption and reduced risk of coronary heart disease: is the effect due to beer, wine, or spirits? British Medical Journal (Clinical Research), 312: 731-736.

11. Hertog MG, Kromhout D, Aravanis $C$ et al. (1995). Flavonoid intake and long-term risk of coronary heart disease and cancer in the Seven Countries Study. Archives of Internal Medicine, 155: 381386.

12. Howard BV \& Kritchevsky D (1997). Phytochemicals and cardiovascular disease: a statement for healthcare professionals from the American Heart Association. Circulation, 95: 2591-2593.

13. Da Luz PL, Serrano Jr CV, Chacra AP et al. (1999). The effect of red wine on experimental atherosclerosis: lipid-independent protection. Experimental and Molecular Pathology, 65: 150-159.

14. Hayek T, Fuhrman B, Vaya J et al. (1997). Reduced progression of atherosclerosis in apoliprotein E-deficient mice following consumption of red wine, or its polyphenols quercetin or catechin, is associated with reduced susceptibility of LDL to oxidation and aggregation. Arteriosclerosis, Thrombosis, and Vascular Biology, 17: 2744-2752.

15. Feng AN, Chen YL, Chen YT et al. (1999). Red wine inhibits monocyte chemotactic protein-1 expression and modestly reduces neointimal hyperplasia after balloon injury in cholesterol-fed rabbits. Circulation, 100: 2254-2259.

16. lijima K, Yoshizumi M, Hashimoto M et al. (2000). Red wine polyphenols inhibit proliferation of vascular smooth muscle cells and downregulate expression of cyclin A gene. Circulation, 101: 805-811.

17. Rosenkranz S, Knirel D, Dietrich $\mathrm{H}$ et al. (2002). Inhibition of the PDGF receptor by red wine flavonoids provides a molecular explanation for the "French paradox". FASEB Journal, 16: 1958-1960.
18. Blanco-Colio LM, Valderrama M, Alvarez-Sala LA et al. (2000). Red wine intake prevents nuclear factor- $\mathrm{KB}$ activation in peripheral blood mononuclear cells of healthy volunteers during postprandial lipemia. Circulation, 102: 1020-1026.

19. Corder R, Douthwaite JA, Lees DM et al. (2001). Endothelin-I synthesis reduced by red wine. Nature, 414: 863-864.

20. Wallerath T, Poleo D, Li H et al. (2003). Red wine increases the expression of human endothelial nitric oxide synthase: a mechanism that may contribute to its beneficial cardiovascular effects. Journal of the American College of Cardiology, 41: 471-478.

21. Chen CK \& Pace-Asciak CR (1996). Vasorelaxing activity of resveratrol and quercetin in isolated rat aorta. General Pharmacology, 27: 363-366.

22. Fitzpatrick DF, Hirschfield SL \& Coffey RG (1993). Endotheliumdependent vasorelaxing activity of wine and other grape products. American Journal of Physiology, 265: H774-H778.

23. Celermajer DS, Sorensen KE, Gooch VM et al. (1992). Non-invasive detection of endothelial dysfunction in children and adults at risk of atherosclerosis. Lancet, 340: 1111-1115.

24. Celermajer DS (1998). Testing endothelial function using ultrasound. Journal of Cardiovascular Pharmacology, 32: S29-S32.

25. Corretti MC, Anderson TJ, Benjamin EJ et al. (2002). Guidelines for the ultrasound assessment of endothelial-dependent flow-mediated vasodilatation of brachial artery: a report of the International Brachial Artery Reactivity Task Force. Journal of the American College of Cardiology, 39: 257-265.

26. Gutierrez MA, Pilon PE, Lage SG et al. (2002). Assessment of carotid diameter and wall thickness in ultrasound images using active contours improved by a multiresolution technique. Proceedings of SPIE, 4683: 248-255.

27. Allain CC, Poon LS, Chan CS et al. (1974). Enzymatic determination of total serum cholesterol. Clinical Chemistry, 20: 470-475.

28. Mano T, Masuyama T, Yamamoto K et al. (1996). Endothelial dysfunction in the early stage of atherosclerosis precedes appearance of intimal lesions assessable with intravascular ultrasound. American Heart Journal, 131: 231-238.

29. Celermajer DS (1997). Endothelial dysfunction: does it matter? Is it reversible? Journal of the American College of Cardiology, 30: 325333.

30. Vogel RA (1997). Coronary risk factors, endothelial function, and atherosclerosis: a review. Clinical Cardiology, 20: 426-432.

31. Flesch M, Schwarz A \& Bohm M (1998). Effects of red and white wine on endothelium-dependent vasorelaxation of rat aorta and human coronary arteries. American Journal of Physiology, 275: H1183-H1190.

32. Andriambeloson E, Stoclet JC \& Andriantsitohaina R (1999). Mechanism of endothelial nitric oxide-dependent vasorelaxation induced by wine polyphenols in rat thoracic aorta. Journal of Cardiovascular Pharmacology, 33: 248-254.

33. Cishek MB, Galloway MT, Karim M et al. (1997). Effect of red wine on endothelium-dependent relaxation in rabbits. Clinical Science, 93: 507-511.

34. Martin S, Andriambeloson E, Takeda K et al. (2002). Red wine polyphenols increase calcium in bovine aortic endothelial cells: a basis to elucidate signalling pathways leading to nitric oxide production. British Journal of Pharmacology, 135: 1579-1587.

35. Stein JH, Keevil JG, Wiebe DA et al. (1999). Purple grape juice improves endothelial function and reduces the susceptibility of LDL cholesterol to oxidation in patients with coronary artery disease. 
Circulation, 100: 1050-1055.

36. Hashimoto M, Kim S, Eto M et al. (2001). Effect of acute intake of red wine on flow-mediated vasodilatation of the brachial artery. American Journal of Cardiology, 88: 1457-1460.

37. Martinez C, Vila JM, Aldasoro M et al. (1994). The human deferential artery: endothelium-mediated contraction in response to adrenergic stimulation. European Journal of Pharmacology, 261: 73-78.

38. Matsuo S, Nakamura Y, Takahashi M et al. (2001). Effect of red wine and ethanol on production of nitric oxide in healthy subjects. American Journal of Cardiology, 87: 1029-1031.

39. Kannel WB \& Ellison RC (1996). Alcohol and coronary heart disease: the evidence for a protective effect. Clinica Chimica Acta, 246: 59-76.

40. Di Castelnuovo A, Rotondo S, lacoviello L et al. (2002). Metaanalysis of wine and beer consumption in relation to vascular risk. Circulation, 105: 2836-2844. 